\title{
Treatment sequence of combined pelvic injury, consisted of upper pubic ramus fracture, sacroiliac joint dislocation with open book injury, using Stoppa approach
}

\author{
Nikolopoulos Fotios, Dimitrios Samaras, Kollintzas Lykourgos \\ General Hospital of Piraeus "Tzaneio», Piraeus, Greece
}

\begin{abstract}
Among pelvic serious injuries is the so-called "open book" injury of the pelvis, with Sacroiliac Joint Disruption (SIJD) in combination with upper pubic ramus or anterior column fracture, contralateral or ipsilateral, or both. This combination of pelvic injury could be classified according Young and Burgess classification as LCIII or CM type (Combined Mechanism) and as 61-B3.1 61B3.2 following AO/OTA classification.

Specifically, the upper pubic ramus fracture can be classified according to Nakatani classification as type I medial of the foramen, type II within the foramen and type III lateral to the foramen. The difficulty to deal with these fractures is how to close and reduct the pubic symphysis in mechanically stable way since there is fracture in one or both the upper pubic ramus. The existence of these fractured elements, in this type of pelvis injury allow a lot of degrees of freedom which must be managed from the surgeon in the proper sequence. The incision and the approach are also mandatory for successfully treating these lesions. Anterior Intrapelvic Approach (AIP) or Stoppa approach in conjunction with the first window of ilioinguinal approach is the most appropriate surgical exposure for reduction and fixation.
\end{abstract}

How to cite this article:

${ }^{*}$ Correspondence to Author:

Nikolopoulos Fotios

General Hospital of Piraeus "Tzaneio», Piraeus, Greece

Nikolopoulos Fotios, Dimitrios Samaras, Kollintzas Lykourgos. Treatment sequence of combined pelvic injury, consisted of upper pubic ramus fracture, sacroiliac joint dislocation with open book injury, using Stoppa approach. American Journal of Orthopedic Research and Reviews, 2021, 4:27.

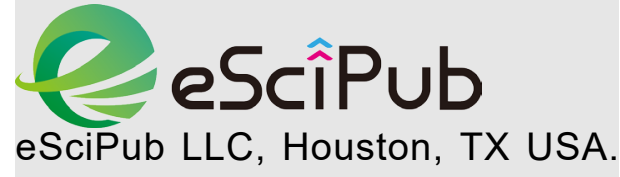

Keywords: Stoppa, approach, anterior, column, pubic, symphy- Website: https://escipub.com/ sis, reduction, fixation, ilioinguinal. 


\section{Introduction.}

Among pelvic serious injuries is the so-called "open book" injury of the pelvis, with Sacroiliac Joint Disruption (SIJD) in combination with upper pubic ramus or anterior column fracture, contralateral or ipsilateral, or both. This combination of pelvic injury could be classified according Young and Burgess classification as LCIII or CM type (Combined Mechanism) and as 61-B3.1 61-B3.2 following AO/OTA classification $[1,2,3]$.

Specifically, the upper pubic ramus fracture can be classified according to Nakatani classification as type I medial of the foramen, type II within the foramen and type III lateral to the foramen ${ }^{[4]}$. The difficulty dealing with these fractures is how to close and reduct the pubic symphysis in mechanically stable way since we have fracture in one or both the upper pubic ramus. These fractured elements of this pelvis injury, allow a lot of degrees of freedom which must be managed from the surgeon in the proper sequence. The incision and the approach are also mandatory for successfully treating these lesions. Anterior Intrapelvic Approach (AIP) or Stoppa approach in conjunction with the first window of ilioinguinal approach is the most appropriate surgical exposure for reduction and fixation.

Typical mechanisms causing pelvic disruption are, traffic injuries $(60 \%)$, falls from a height $(30 \%)$ and crush injury under heavy weights $(10 \%) .{ }^{[5]}$
The posterior $\mathrm{SI}$ ligament is a key vertical stabilizer, maintaining the sacrum in its normal position in the pelvic ring ${ }^{[6]}$.

The pubic symphysis is stabilized by a series of ligaments and primarily serves as a strut to improve anterior ring stability during ambulation. However, the pubic symphysis is the weakest link in the pelvic ring, contributing only $15 \%$ of intrinsic pelvic stability ${ }^{[7,8]}$.

In general, pelvic stability is achieved via the ability of the osseoligamentous structures to withstand physiologic stresses without abnormal deformation.

In this three-case report study we examine the reduction and fixation sequence of the above complex pelvic injury.

\section{Cases presentation:}

First case is a man 55 years old with an open book injury combined fracture in both upper pubic rami and Sacroiliac joint (SIJ) diastasis. Especially the right side of the rami is Nakatani I and the left side is Nakatani III, and the left SI joint is widely opened needing reduction and fixation. The left sided hemipelvis is also rotated and stands in unequally height compared the right hemipelvis Fig 1 suggesting that is not only an LCIII injury according Young-Burgess classification but is a Combined mechanical injury (CMI) due to the vertical shearing component of the left side hemipelvis.

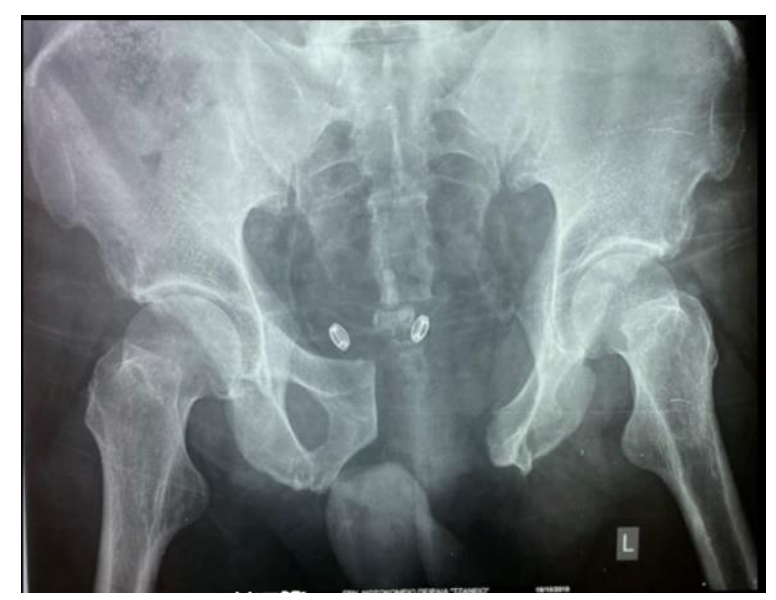

Fig 1. Case 1. Open book injury, CMI according Young-Burgess classification. Notice the left hemipelvis is malrotated and in unequally height in comparison with the right hemipelvis. 
Of course, there are also fractures in both ischial Initially, an external fixation (ex-fix) has been put tuberosities Fig 2, which is evidence that the in supracetabular position Fig 3, to turn and sacrotuberous ligaments especially left sided is reduct the left wing. incompetent.

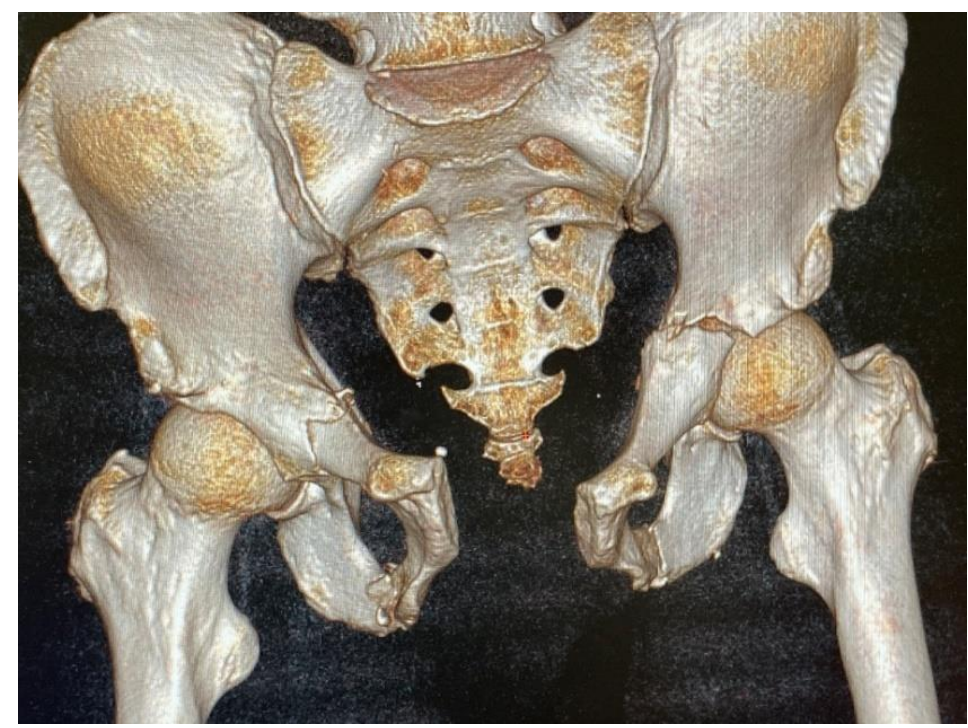

Fig 2. Case 1. Both ischial tuberosities are broken. Notice the malrotation of the left hemipelvis \& the $\mathrm{SI}$ joint both widened, especially in the left side. Also notice the upper

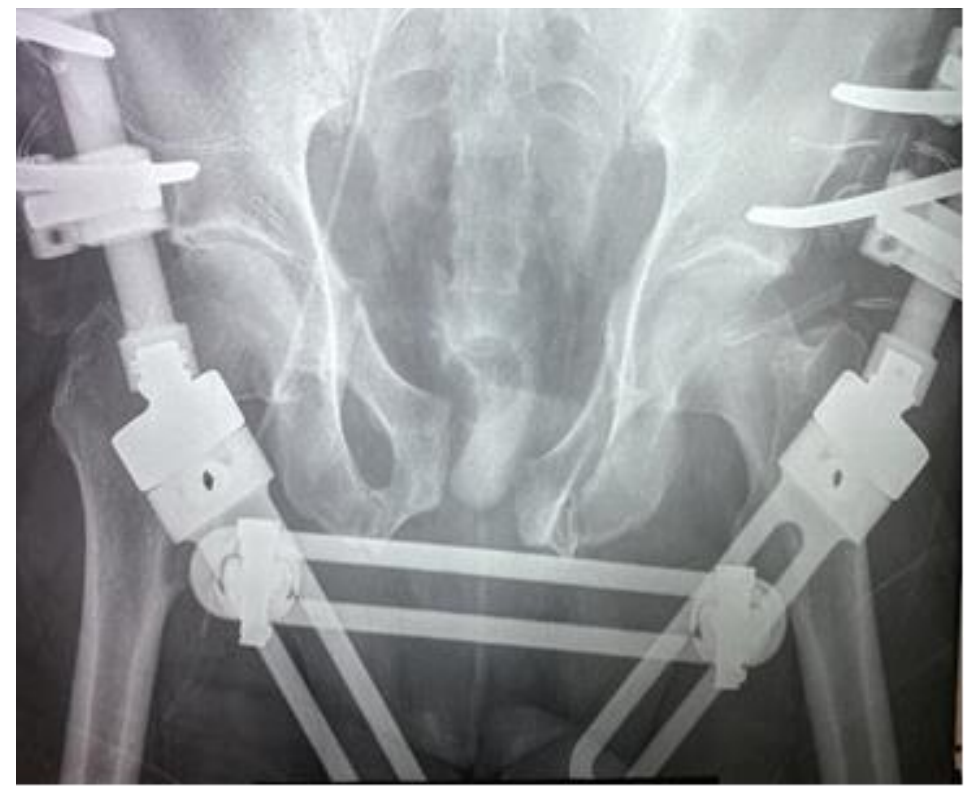

Fig 3. Case 1. Initially an ex-fix was put to stabilize and reduct the pubic sym-physis \& the left iliac wing. It was not possible to perform the reduction because the left wing was hitched on the sacrum posteriorly.

Then, the sequence of the reduction and fixation was the following: Firstly, we stabilized the two rami fractures. This has been accomplished using a plate in the left side and a retrograde screwing to the right pubic rami via Stoppa (Anterior Intrapelvic Approach). Then, after redacting, closing and fixing the pubic symphysis with the use of a plate via the same approach, we closed and fixed the left SIJ widening Fig 4, using also a plate anteriorly, via the first window of the ilioinguinal approach. 


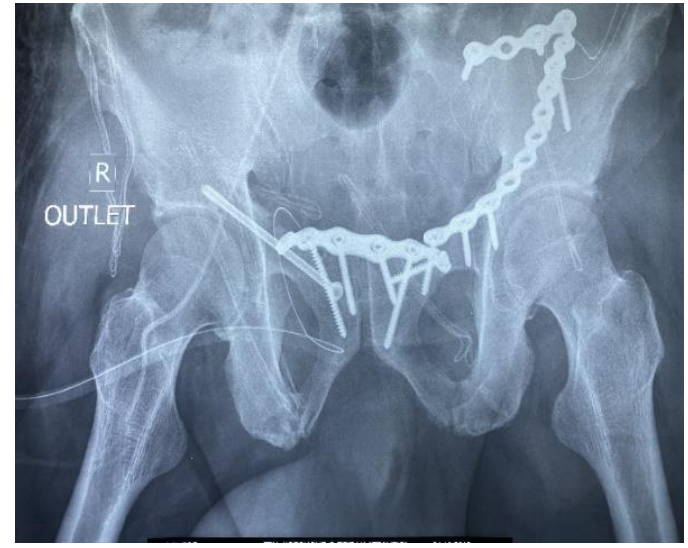

Fig 4. Case 1. In the outlet view (above) the retrograde screw right \& the plate left were placed firstly.

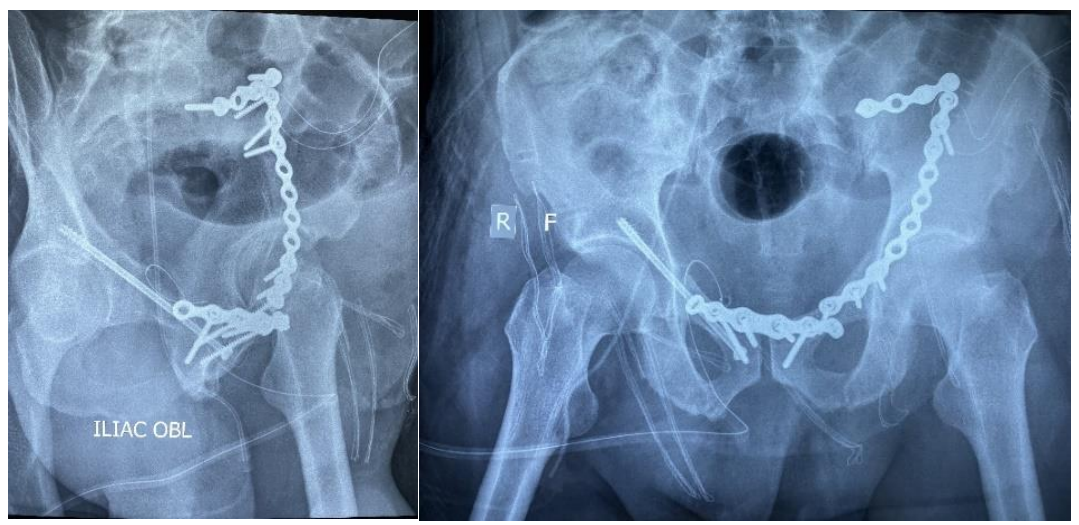

Fig 4. Case 1. Notice the perfect reduction of the pubic symphysis and SIJ fixation in face projection. In the iliac oblique view projection, we con-firmed the final position of the retrograde screw.

Three weeks postoperatively the patient was able to walk with crutches partially weight bearing.
Second case is almost like the first case. Only difference is the left sided fracture contralateral to the sacrum injury.
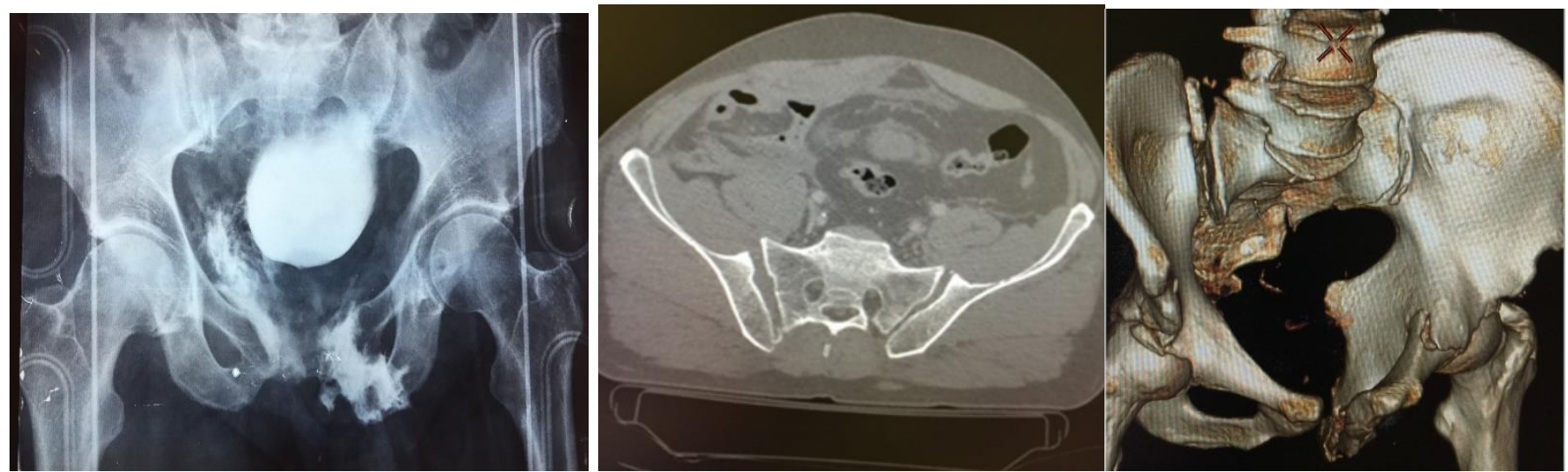

Fig 5. Case 2. Open book injury in combination with and SIJ disruption right side and rami fracture Nakatani II in left side. Left: Anteroposterior x-ray, there was also a bladder rupture as the contrast agent diffuses in all the true pelvis. Middle: CT/scan-notice the SIJ disruption in the right side. Right: 3D/reconstruction, notice the upper pubic ramus fracture on the left and the SIJ disruption in the right side. 
Before closing the pubic symphysis, we must the symphysis to succeed a satisfactory firstly fix the upper pubic rami in a very stable reduction. Even though we applied through the way. The reason we do this, is because, in the reduction forceps large scale forces, there was attempt of closing the symphysis, a great deal of great deal of difficulty in reducing the symphysis. forces will be developed, enough to displace the Finally, two plates in almost $60^{\circ}$ each other were rami fracture. So firstly, we put a plate, in the placed to hold the reduction in the SIJ, using the upper pubic rami using the Anterior Intrapelvic first window of the ilioinguinal approach.

Approach (modified-Stoppa Approach).

Secondly, a separate plate has been placed in

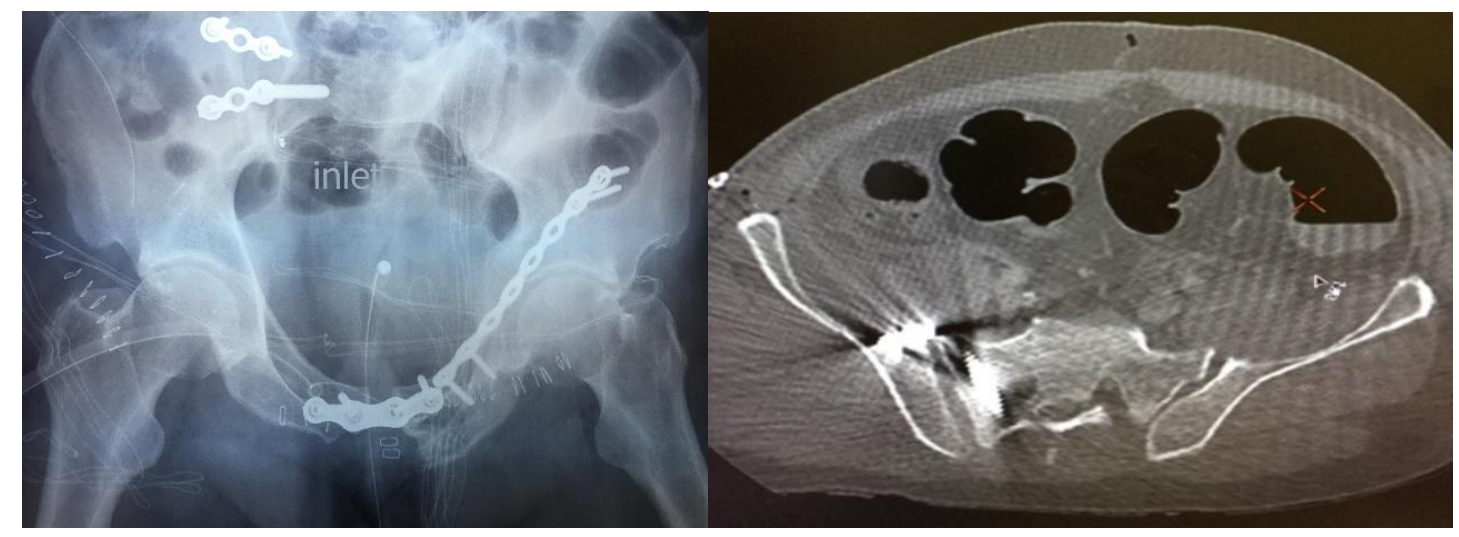

Fig 6. Case 2. Right: Four plate were used. Firstly, the right pubic rami stabilized with a long plate, secondly the pubic symphysis secured with a strong special plate (Stryker) and finally the two plates were set in the SIJ. The reduction-closing of the SIJ, using forceps presupposes the stabilization of the symphysis. Left: A postoperatively CT/scan confirms the good position of the $6 \mathrm{~mm}$ screw which used through the plate since passed beside the right sacral foramen leaving it free.

As you can see in Fig 7, the x-ray 6 weeks Stoppa in conjunction with the upper two postoperatively (p.o) the reduction was stable. windows of the ilioinguinal approach.

The approach which was used was the modified
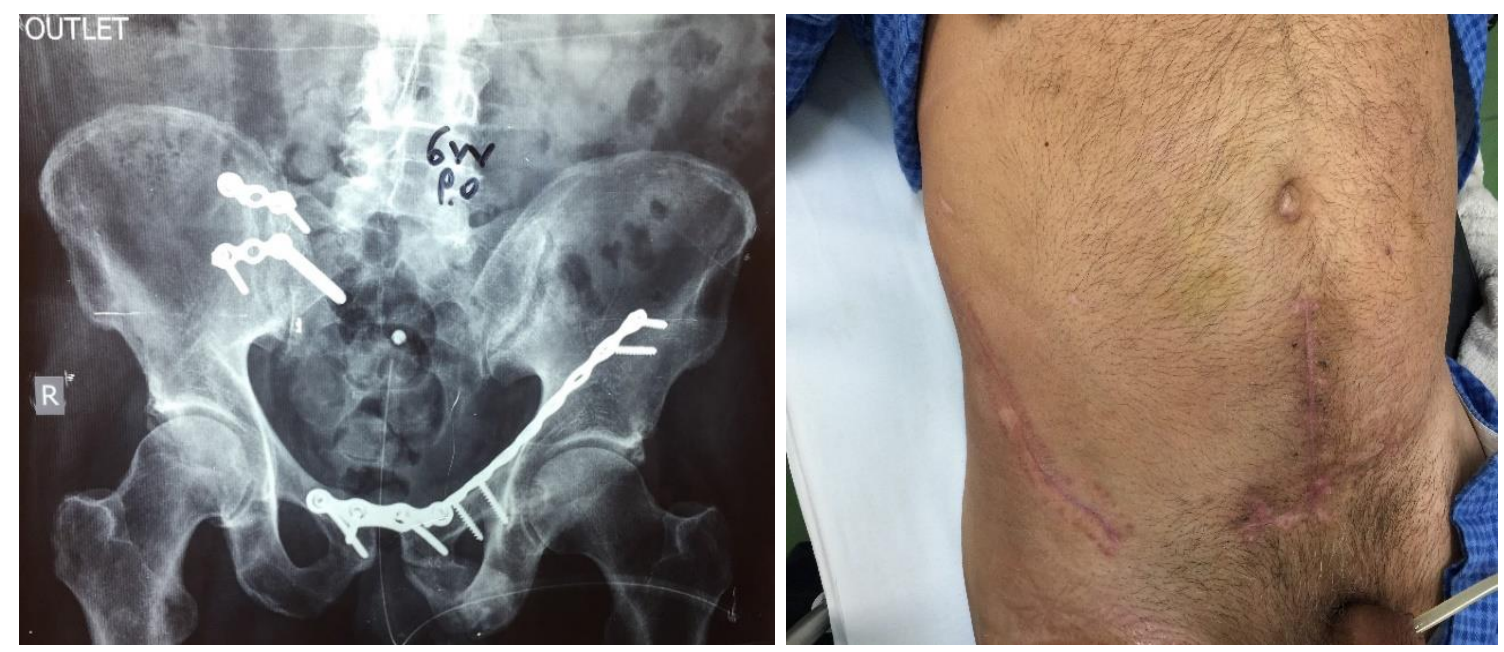

Fig 7. Case 2. Left side: 6 weeks an anteroposterior $x$-ray reveals stable osteo-synthesis. Right side: The approach was used is the modified Stoppa in con-junction with the upper two windows of the ilioinguinal approach. The vertical limp of the Stoppa approach to the umbilicus through linea alba helps us to re-veal the upper pubic rami until the iliac to secure the osteosynthesis with the plate. 
The x-ray 16 months postoperatively, depicts Fig malposition of the symphysis, does not impair 8 , the residual malposition of the pubic the functionality of the patient and he is symphysis due to the difficulty we had to incredibly happy because walks and sits without manipulate and reduct it. The existence of this pain or other disturbances.

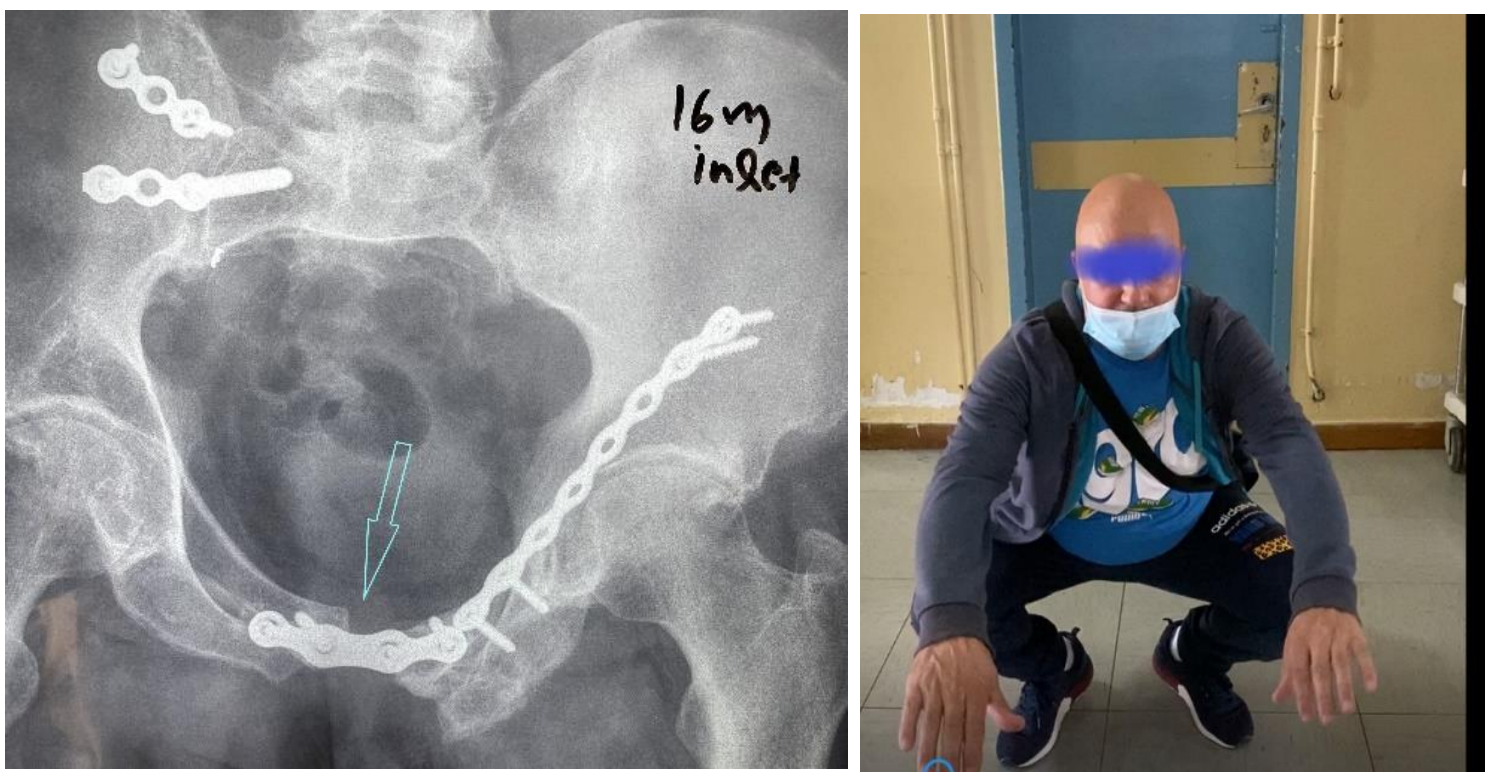

Fig 8. Case 2. Left side: The x-ray $16 \mathrm{~m}$ postoperatively depicts the residual malposition of the pubic symphysis due to the difficulty we had to manipulate and reduct it. Right side: Even though the existence the malposition of the symphysis the patient is incredibly happy because walks and sits without pain or other disturbances.
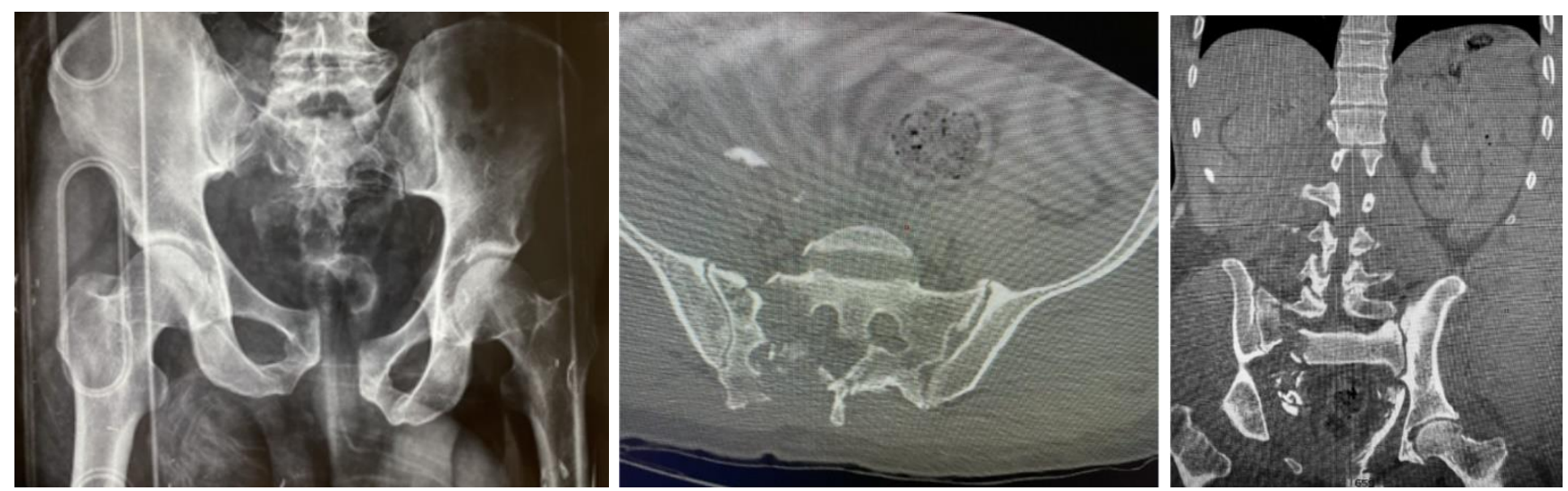

Fig 9. Case 3. Left side: The x-ray depicts the open pubic symphysis and differ-ence in height of the hemipelvis. Something happens in the sacrum. Middle: Sagittal CT/scan reveals a comminuted fracture of the sacrum without SI disruption. The right sacrum is displaced due to the fracture. The patient was unstable. There was not neurological deficit because the sacral fracture is Den-nis II, so the nerve roots were not impaired. Right side: Coronal CT/scan view, notice the comminuted right side of the sacrum.

Third case is a labor who fell from a tree 7 meters high. The patient in case 3, had CM (Combined Mechanism) pelvic injury Fig 9, according Young and Burgess Classification of pelvic Ring Injuries.

The patient was hemodynamically unstable. In the framework of damage control injury, the 
patient was lead in the OR for positioning a C- anterior supracetabular external fixation put clamp for posterior stabilization Fig 10 and an immediately.
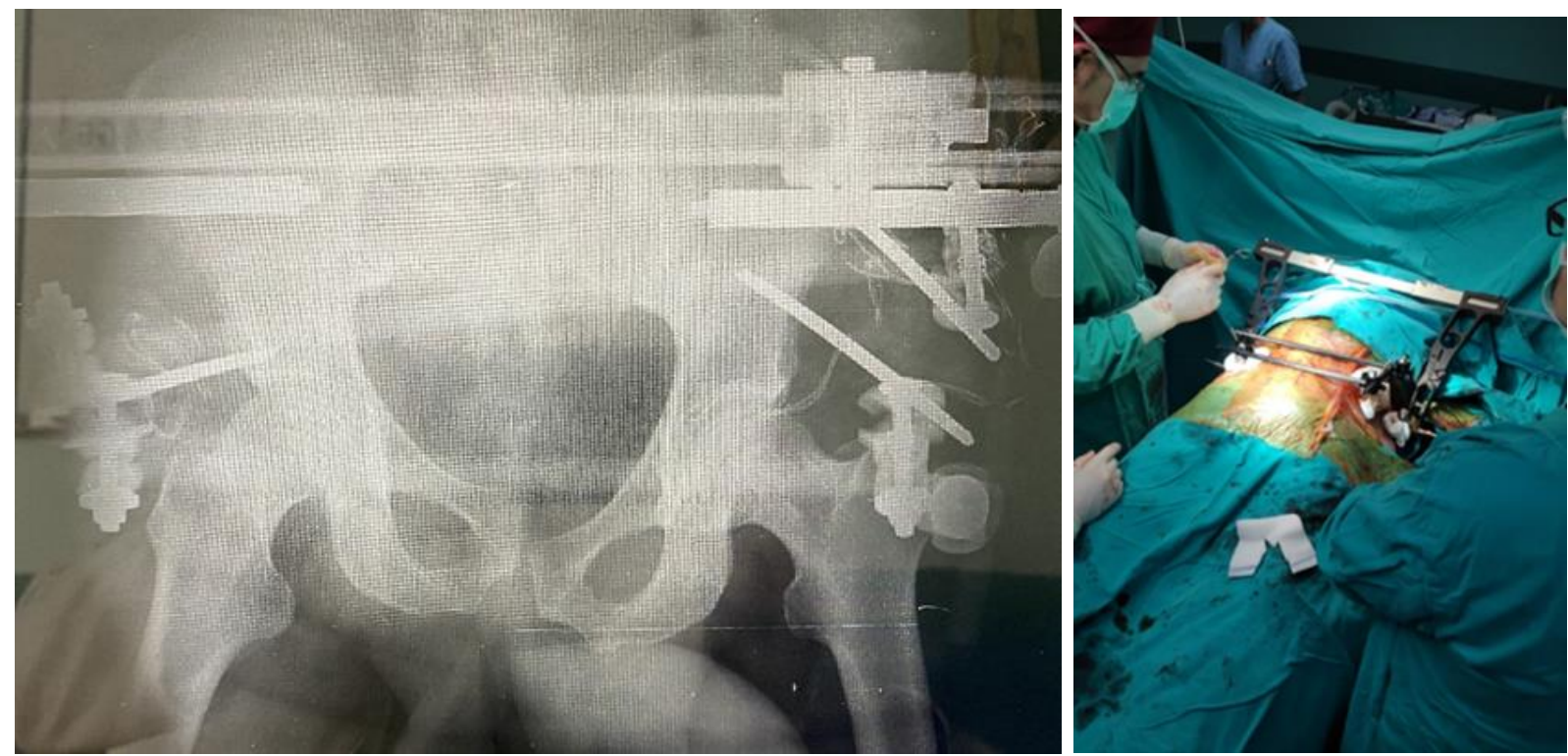

Fig 10. Case 3. Left side: Postoperatively x-ray with the C-clamp \& the External fixator in positioning. Right side: Intraoperatively, the two external fixators in place

Notice in Fig 10 that, despite the two external fixators and the efforts that had been made to reduct the right hemipelvis, this was unfeasible. The malreduction remains. The main purpose of course, for the use of the external fixators was to stop the bleeding and not to reduct the fractures.
A few hours later the patient became in stable condition.

A few days later, the patient was lead again in the OR for the final operation. A plate was put in pubic symphysis Fig 11 and a sacral posterior plate at the sacrum.
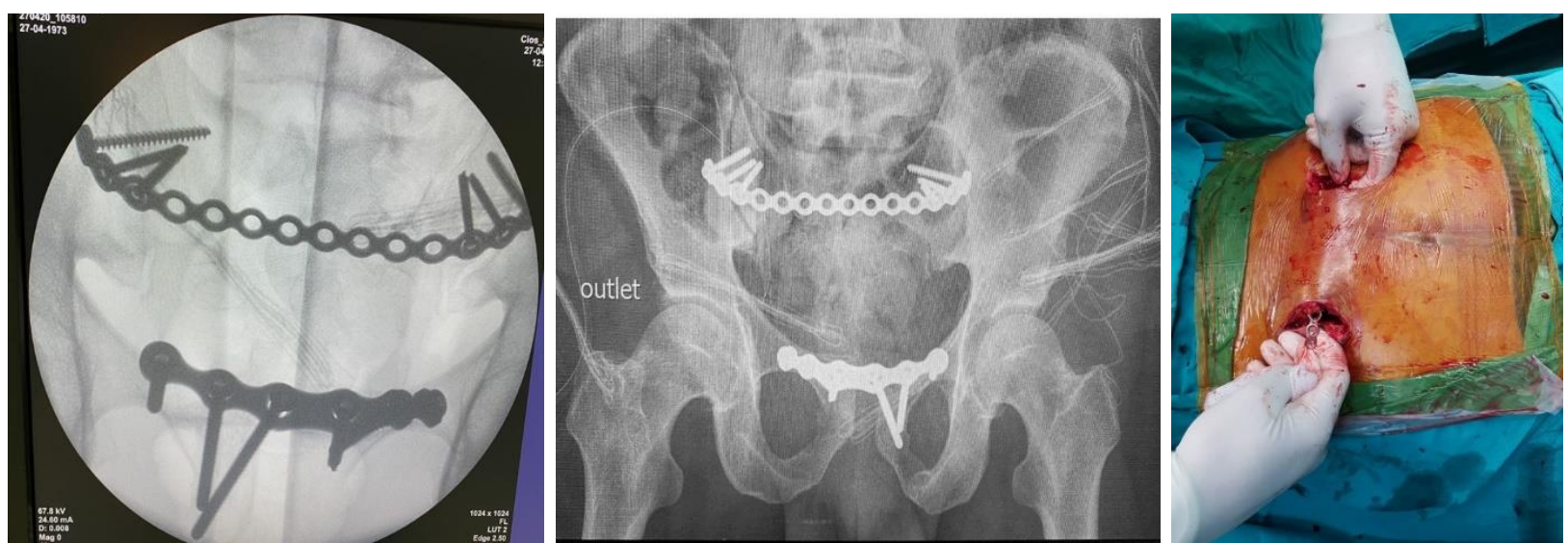

Fig 11. Case 3. Left: intraoperatively the c-arm view, plates in good positioning. Middle: the outlet view postop x-ray. Right: Intraoperatively photo depicts how the plate is placed to the posterior sacrum.

The postop $x$-rays reveal not satisfactory reduction of the symphysis. Although there were not so accurate reduction of the symphysis, we considered that it would not be a problem for the patient so we didn't revise the osteosynthesis. 

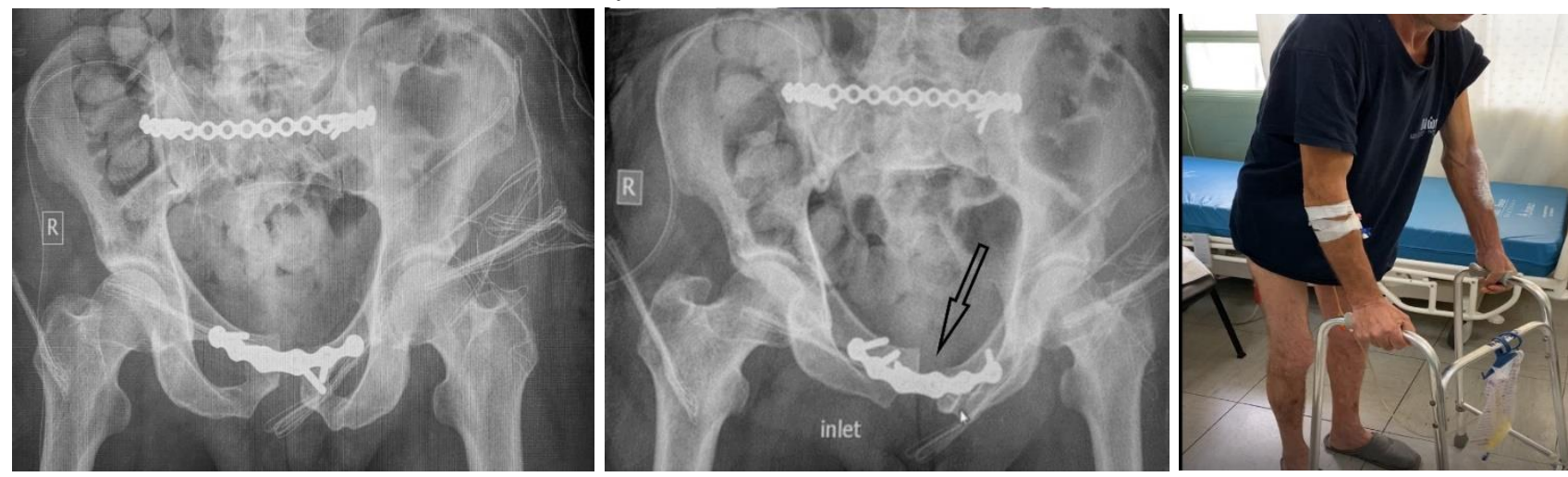

Fig 12. Case 3. Left: Postop x-ray, anteroposterior view demonstrates inade-quate reduction of the right hemipelvis. Middle: the inlet view depicts incon-sistency of the pubic symphysis (arrow).

\section{Discussion:}

In the first case, eventhough, we put initially an ex-fix, it was not possible to accomplish reduction of the symphysis. As you can see, the ex-fix screws bent under the applied forces to reduct the symphysis. That was due to the hindrance of the iliac wing to the sacrum. Fortunately, notice the perfect reduction finally. This was accomplished easily with the pubic symphysis plate. On the other hand, the reduction of the symphysis had great deal of difficulty in case 2 and in case 3 . In the last two cases the unsatisfactory symphysis reduction, was due to indentations of the sacral fracture and/or the small fragments, which provoke obstacles and prevent the accurate reduction. A solution to this might be the ORIF (Open Reduction Internal Fixation) of the sacral fracture. However, in my opinion the disadvantages of this effort outweigh the advantages because the fracture manipulations and the skin impairment needed, will create more problems than solutions. Additionally, when we reduce the pubic symphysis, we can turn the patient more safely in prone position, to reduct the sacrum displacement and fixing the sacrum from posteriorly as the final stage of the sequence.

Summarizing, the sequence of this kind pelvic injuries was as follows: firstly, the anterior column/the pelvic brim/rami fracture, then the pubic symphysis and finally the sacrum. This sequence of actions seems to give more convenient and successfully results.

\section{Conclusions:}

It's a big challenge to cope with such complicated fractures. The advice we can share, are the following: Operate as soon as possible, fix first the anterior column, in one or in both sides, using the most familiar approach but especially when you have low or middle anterior column fractures, use Anterior Intrapelvic Approach. Then, close and fix as stable as you can the pubic symphysis and finally, use the first window of the ilioinguinal approach, to fix the Sacroiliac joint or perform cannulated transcutaneous sacroiliac screwing.

\section{References}

[1]. AOTRAUMA, "Fractures of the Pelvis and Acetabulum, Principles and Methods of Management-Fourth Edition" Marvin Tile, David L Helfet, et al. Vol 1-Pelvis, 2015, Table 1.3-3, p.53

[2]. "Rockwood and Greens's Fractures in Adults" Paul Tornetta et al, Ninth Edition,2020 Wolters Kluwer, Vol 2, Section three, "Axial Skeleton, Pelvis and Acetabulum" Table: Young and Burgess Classification, P.1976

[3]. AOTRAUMA, "Fractures of the Pelvis and Acetabulum, Principles and Methods of Management-Fourth Edition" Marvin Tile, David L Helfet, et al. Vol 1-Pelvis, 2015, Table 1.3-1, p.49

[4]. AOTRAUMA, "Fractures of the Pelvis and Acetabulum, Principles and Methods of Management-Fourth Edition" Marvin Tile, David L Helfet, et al. Vol 1-Pelvis, 2015, Fig 1.3-18 
"Nakatani classification of superior pubic ramus fractures"

[5]. Schmal H, Markmiller M, Mehlhorn AT, Sudkamp NP. Epidemiology and outcome of complex pelvic injury. Acta Orthop Belg 2005;71(1):41-47. Medline, Google Scholar

[6]. Kellam JF, Mayo KA. Pelvic ring disruption. In: Skeletal trauma. 3rd ed. Philadelphia, $\mathrm{Pa}$ : Saunders; 1052-1108. Google Scholar

[7]. Durkin A, Sagi HC, Durham R, Flint L. Contemporary management of pelvic fractures. Am J Surg 2006;192(2):211-223. Crossref, Medline, Google Scholar

[8]. Kurylo JC, Tornetta P 3rd. Initial management and classification of pelvic fractures. Instr Course Lect 2012;61:3-18. Medline, Google Scholar 